\title{
The risks of using continuous deep palliative sedation within the context of euthanasia
}

\author{
R. Polaks \\ University of Latvia, Riga, Latvia
}

\begin{abstract}
Although palliative care is one of the main arguments among the opponents of euthanasia, the individual medical activities implemented within it are not always evaluated unequivocally. Considering that patient in such care centres arrives mainly at the last stages of the disease when intensive treatments are no longer able to help, to reduce discomfort and relieve pain caused by the disease, analgesic means can be used that can shorten the patient's life expectancy and cause death. Such undesirable consequences can be seen in the deep and continuous palliative sedation, which not only is the last resort for pain prevention process, but also is still quite debatable medical and legal doctrine, seeing in it a similarity to the so-called "easy death", resulting in an unofficial name - "slow euthanasia". It is therefore important to emphasize that deep and continuous palliative sedation is considered medically correct action only if its application is justified by the need to relieve the incurably ill person from the grievous pain and sufferings caused by the disease, not to cause death, and only when in certain clinical circumstances, it cannot be achieved by other means and methods. In all other cases, depending on the state fact matters, activities of a physician constitute either an active voluntary or non-voluntary euthanasia, which in most countries of the world is a subject to criminal sanctions.
\end{abstract}

Key words: Active voluntary euthanasia, active non-voluntary euthanasia, continuous deep palliative sedation, "double effect principle", approbated procedures in medical theory and practice

\section{Introduction}

Although palliative care is one of the main arguments among the opponents of euthanasia on the grounds gained in practical reasons, the individual medical activities implemented within it are not always evaluated unequivocally. Palliative care of incurably ill people is extensive and, at the same time, a complex set of measures, ranging from social issues and, finally, to psychological preparation of family members as well as incurably ill persons themselves to death. However, relieving of pain and sufferings of incurably ill people is among its most important tasks, given that palliative care takes place within the treatment process. A patient arrives in such care centres mainly at the last stages of the disease, when intensive treatments are no longer able to help. Analgesics used to reduce discomfort and pain caused by the disease can shorten the patient's life expectancy and cause death [1]. Such undesirable and, at the same time, inevitable consequences can be seen in medication overdose, as well as within palliative sedation when it is deep and continuous, or as it is also described - terminal, which are still sufficiently controversial issues in medical and legal doctrine, being similar to euthanasia. Thus, in the context of "easy death" issue it is important to delimit the activities that deliberately focus on causing of death of incurably ill person with the aim, out of compassion, to release disease-induced grievous pain and sufferings, or euthanasia, of 
the activities identified as 'normal medical practice' in the care of such patients, although the realization consequences could be associated with the patient's life expectancy reduction or premature death. Absence of marked borderlines for decades has contributed to a false idea of ??what is the maximum means or method in incurably ill patient care, and under what circumstances they should be attributed not to euthanasia, but rather to standard medical practice, taking into account the fact that the risk level of the practicing hidden euthanasia under the guise in pain reduction or prevention is quite high.

\section{Materials and methods}

Analytical, comparative, inductive and deductive, as well as logical scientific research methods were applied in order to achieve the aim of the article, in the course of deep and continuous palliative sedation theoretical framework and its practice.

\section{Results and discussion}

Medicinal products, used in medical care of incurably ill patients, whose unwanted side effect is associated with the person's life expectancy reduction or death, is not the only controversial issue within the principle of double effect. The subject to critical analysis is also palliative sedation when it is deep and applied continuously, causing the patient's coma to the condition leading to death, announcing as the basic aim the release of the patient from the grievous pain and sufferings caused by the disease, or, as it is defined by Stephen W. Smith, when the applied medication causes coma-like condition to the patient [2].

The attitude towards the use of deep and continuous palliative sedation in care of incurably ill people, when, for the purposes to prevent sufferings, the patient has been sedated dead to the world until death occurs, for many years in medical and legal doctrine has been different, mainly by extending the discussion on the current Dutch practice of terminal care of patients. For example, in the Netherlands, even quite recently, i.e., in 2003, among doctors and lawyers an understanding of what is ante-mortem medical intervention standard practice in treatment of incurably ill patients, if it leads to the patient's death, was different. The President of the Board of Prosecutors General has stated that sedation of incurably ill person who is experiencing unbearable sufferings to the point that the patient has become unconscious, can be justified from the medical point of view, but from the legal point of view it can still be regarded as life termination [3]. This is why for many years in the Netherlands it was considered as one of the independent forms of euthanasia [4], which relatively limited the intensity of its practice.

With the aim to harmonize the medical and legal nuances of the test subject and to delimit deep and continuous palliative sedation from the activities of termination of life of the incurably ill person, or euthanasia, in 1849 was founded the Netherlands Medical Association (Koninklijke Nederlandsche Maatschappijtt bevorderingder Geneeskunst (KNMG)) that in 2005 published the "Guideline for Palliative Sedation", with subsequent amendments carried out in January 2009[5], declaring it not as a response to the effects of the given period, but as the solution in the context of palliative care.

According to the "Guidelines for Palliative Sedation" with "palliative sedation" is meant intentional lowering of patient's consciousness level on their last stages of life with a view to decrease sufferings that can be achieved in two ways: with continuous deep sedation, effect leading to the patient's death, which is always applied for terminal patients experiencing unbearable sufferings and, where it is foreseeable that their death is inevitable in one or two weeks and with the temporary or persistent sedation. 
Special dispute in the context of euthanasia is caused by deep and continuous palliative sedation in particular requiring a thorough analysis of the sedative effect.

As stated in the Guideline, palliative sedation obtains medical excuse in cases where one or more symptoms inevitably cause unbearable sufferings and if none of the medical treatments has been effective enough or have caused unwanted side effects. By contrast, in the document, with the sufferings is understood not only physical pain, psychosis and acute shortness of breath, but also "existential sufferings", which are classified as difficult to treat medical symptoms that cannot be eased with the communication and mental assistance. In cases of existential sufferings, typically observed in severe diseases, patients are weakened and close to death, expressing complaints about the physical pain. Since the feeling that further existence has lost its importance can also cause unbearable sufferings, patients have the right to the last moments of life when death is expected in the coming week or two to ask the doctor to apply continuous sedation. Considering that the concept of "existential sufferings" has been widely interpreted additionally to medical ones, the Guideline includes a clause on the necessary opinions from psycho-sociological and mental problem experts, confirming that suffering is indeed unbearable.

Subject to the requirement that a deep and continuous palliative sedation is considered a medical procedure, the responsibility for medical symptom detection, decision making and its execution in all cases lies with the physician irrespective of the fact that it should be based on the mentally capable patient's informed consent, while in its incapacity cases a physician is consulting with the representative. This principle is provided for only two exceptions: the doctor may disregard rejection of the incapable patient representative for the application of deep and continuous sedation, unless it is done in the patient's interest and in acute situations in the sense of a sudden patient's deteriorating state of health, causing unbearable sufferings, when the doctor has no opportunity for immediate consultation with the patient or their representative.

Decision making on application of deep and continuous palliative sedation may be affected by a number of factors - the doctor and the patient's perception of a "good death", the importance of the symptoms and their quantity, somatic pain impact on the patient's well-being and anxiousness, fear of death and its processes, helplessness, sorrow, anger, sadness, disease duration, etc. Even physical fatigue can affect decision-making and promote sufferings. For the decision to be recognized as a legitimate and well-founded, in the interest of its transparency, a physician is required to document deep and continuous palliative sedation process, stating the reasons why this method was chosen, the information about the patient's medical condition, the way the patient was sedated, what means were used and the like.

As it has been highlighted above, deep and continuous sedation in the Netherlands is applicable only in those cases when it is foreseeable that the patient's death will occur in the coming week or two. However, given the fact that it is not always possible to pinpoint the likely time of death, the physician, according to the Guideline, should focus not on the exact timing of the death, but on progress of the disease, concluding that the patient is really dying. However, opponents of such overly broad interpretation can be found. Govertden Hartog states that in those cases when sedation is launched before the time when patient's life expectancy is longer than one or two weeks, the following actions should always be seen as a form of euthanasia [6].

Although Guideline for deep and continuous palliative sedation recommended the medicament midazolam, whereas the use of opioids and other means that are not intended as sedatives, but are sometimes used to achieve this effect, is considered as a wrong practice, however, this rule is not always observed. Foreign literature also indicates, although the 
use of high doses of opioids and terminal sedation is considered to be standard procedure in palliative medicine to facilitate symptoms of an incurable disease throughout the death process, these can contribute to causing the death [7]. Also, barbiturates, which represent a significant "aggressive step" in the treatment process, create a risk of causing a life-shortening effect [8]. Therefore, as Penney Lewis indicates, abstaining or stopping saving lives and providing patients with analgesics in adequate quantity all together may shorten life span or accelerate death [9].

Special attention in the issue of deep and continuous palliative sedation should be paid to the indication expressed in Guideline that, within the framework of its execution, artificial provision of hydration of the patient is always stopped, regardless the fact whether, before it starts, the patient has or has not been able to pick it up on their own. The necessity of withholding of artificial hydration is based on a number of practical and medical reasons, namely: the following activities should be considered as useless, since basing on the principle of proportionally involved resource utilization, they exceed the potential benefits from treatment, realizing that the patient has incurable morbidity causing grievous sufferings, and its consequences, i.e., death is inevitable; initiating artificial hydration can prolong the duration of the sufferings, or even intensify them, increasing oedema, bronchial contents, urine promotion, etc.

As stated in the Guideline, keeping to the provision that the patient at start-up time of deep and continuous palliative sedation is actually dying, it is unlikely that the patient will die sooner if they are not provided with artificial hydration and hence, in all such cases it is presumed that the patient actually dies of the disease, and not due to dehydration.

Anticipating that the deep and continuous palliative sedation can lead to a range of ethical, medical and as well legal issues, seeing the actual similarity to euthanasia, at the end of the Guideline there is an Annex with the list of the mutual differences. Although it is possible to agree with the majority of the delimitating criteria, some of them cast doubt on their validity.

As indicated in the Annex to the Guideline, the sedation goal and the means of its achieving is by reducing the sense to release the patients from sufferings, while euthanasia aims to put to end sufferings, terminating the life. This criterion draws our attention to the fact that, although in the Guideline are used different terms, i.e. "liberation from sufferings" and "suffering termination", both the aim of deep and continuous palliative sedation, as well as euthanasia in fact is identical to release incurably ill people from disease-induced grievous pain and sufferings. The only difference that exists between these unusual methods is that in deep and continuous palliative sedation cases, this is achieved by the patient resulting in a deep state of unconsciousness, while in the case of euthanasia - causing death. As deep and continuous palliative sedation process is suspended at the moment of the incurably ill person's time of death, and by then in most cases it is an ongoing process, resulting from its essence presumed that its primary task is to ensure that patient from its inception until death does not longer feel grievous pain and sufferings caused by disease, to facilitate its withdrawal, so the indication of the objective differences is not legally accurate. That knowledge indirectly confirms the guidance indicating that a deep and continuous palliative sedation is often seen as an alternative in the cases when the patient does not want to experience sufferings, but also does not want to them to be used assisted suicide or euthanasia, or in cases where the prerequisites for both methods in probability are equivalent the patient should decide if they want or do not want to save their consciousness until the time of the death.

As the last ambiguously assessed difference in the Annex to the Guideline is that deep and continuous palliative sedation, unlike euthanasia, does not shorten the patient's life expectancy. 
Presumably, no doubt will claim the statement that the human body is able to function if daily nutrients and, in particular, liquid is received. In any case, such mean deliberate interruption in the nearest period of time will inevitably lead to human death. Certain progress of the disease can contribute to such an important body substances, either natural marginalization, or the patient's inability to accommodate them independently that in everyday treatment process is normally removed by artificial methods. Therefore currently the medical and law framework recognizes that an artificial provision of organism with nutrients and fluids is a medical method of treatment, depending on the request, it may be suspended [10], although the question of whether such activities fall under the term "medical care" prompted a series of considerable discussion in the XX century [11]. Abstracting from any possible medical practice occurring situations when artificial provision of organism with nutrients and fluids is interrupted as a necessity in the light of medical indications, and such situations applying exclusively to deep and continuous palliative sedation, it is important to ascertain whether such operations are always medically justified and death to be deemed to be natural, or on the contrary to be intentionally induced.

At present, mainly there are two opinions as to whether refraining from artificial hydration and feeding contribute or not the patient's death. The statement that when practitioners cease artificial hydration and feeding application, thus speeding up the patient's death [12], the opponents argue that the death in such cases is actually caused by another reason, which prevents the patient from eating and drinking [13], i.e., as the result of the disease. In this context, we should tend to think that in artificial feeding and hydration termination case, death is the result of the disease rather than of the operations. However, legally more complicated situation is resolved in cases where the patient retained the ability independently absorb nutrients and fluids, and it is interrupted for the reason that it requires a particular medical procedure, and in the context of analyzed theme - deep and continuous palliative sedation.

The main task of palliative care and, at the same time deep and continuous sedation, is to maintain patient's comfort and dignity during the last moments of their life, rather than with all possible means to prolong their life. Stopping of artificial feeding and hydration if it is determined by medical indications, the patient's health status may deteriorate or new sufferings will be caused, or already existing sufferings will be extended, etc., all cases are medically and legally justifiable. Ethically and legally more complex judgments are subject to situations where the feeding and hydration are discontinued "forcibly" individually or are combined with sedatives, i.e. cases where it is justified by the specifics of the method or the continued use uselessness, such as now in the Netherlands. So legal scholar Emily Jackson once highlighted, although it is accepted medical practice, palliative sedation in some cases, however, may be very similar to euthanasia [14].

The question of whether the termination of hydration individually or in combination with sedatives, promotes the death of a patient, in the doctrine is still valid today. As the Hartog, G. indicates, deep and continuous palliative sedation is mainly realized through the use of such drugs like benzodiazepines, especially midazolam, which is devoid of life-shortening effect, not only when it is used due doses nor overrun, thus the sedation itself does not shorten human biological life [15]. Also, in cases where sedatives were combined with terminated hydration and the patient died in the next few days, in the Netherlands consider that it was caused by a disease, not dehydration, because its suspension is just a way to let die, whereas in cases where the death duration is greater, will be opponents, who believe that terminal sedation combined policy and refraining from hydration has contributed to the patient's life reduction, and if it was carried out on the basis of the patient's request, it is euthanasia [16], and such are not a few [17]. Although Hartog, G. disagree with this statement and stresses that these actions are not homicide, nor any of the forms of euthanasia, yet he recognizes that, from the 
second week, it may no longer be possible to determine the cause of death, there is a strong probability that death even partly occurred due to dehydration [18].

It is not permissible to ignore the fact that the cessation of hydration that is not justified by medical indications and in any case contributes to the individual organism viability reduction. Also unacceptable in deep and continuous palliative sedation is considered any activity that reduces the patient's life expectancy or causes death, which is clearly contrary to the palliative care mission - not to shorten or prolong the patient's life expectancy. Therefore in all cases, when hydration cessation was not based on medical indications and contributed to the decline of patient's life expectancy, being in a direct causal link with the consequences, i.e. the patient's death, the following doctor's actions are not recognized as a deep and continuous palliative sedation and do not apply to allowable medical care, but is classified solely as the deliberate homicide of a person out of compassion, or active euthanasia, which should continue to be viewed in the context of criminal law. In such cases, depending on the will of the person, subject to the euthanasia act, or on the contrary, in the absence of that before the initiation of the procedure, which will give rise to the person's death, to talk to either the active voluntary euthanasia in the sense of where the basis of the incurably ill person's explicit request which in any way and by any means made in a reasonable time before the commencement of euthanasia act, a physician or any other person, by compassionate motives in a manner that does not cause additional pain and sufferings, with their active operations directly cause death of incurably ill person with the aim to release them from the grievous pain and sufferings caused by the disease, or for the active non-voluntary euthanasia, when a physician or any other person out of compassion, with the aim to release from the grievous pain and sufferings caused by the disease through active actions which do not create additional pain and sufferings, cause death of incurably ill person, without clarifying their will of the permissibility of the use of such method against them, considering that this person has not previously stated the will, but now is no longer able to do so.

Supposedly, among the most important arguments that a deep and continuous palliative sedation actually delimitates from any type of euthanasia should be mentioned the fact that in euthanasia cases the performer intended to relieve the incurably ill person from the grievous pain and sufferings out of compassion, intentionally causing or not prolonging the illness, caused natural death moment, while in the sedative effect cases a person applying it also allows shortening of the patient's life expectancy, but in cases of sedative effect the applier, although allows shortening of the patient's life expectancy, in fact does not want such consequences and death as the result of their operations is natural. Therefore, in cases where a deep and continuous palliative sedation is used solely in accordance with its objectives, it is not recognized as an independent form of euthanasia, but the acceptable medical practice in the care of incurably ill people. A similar view also finds justification in foreign legal doctrine, where it is believed that if the palliative sedation is applied proportionately, also deep and continuous in order to achieve the necessary control of the pain, it cannot be equated with the termination of life [19].

Considering that palliative sedation, also deep and continuous, shall be deemed legal and at the same time last resort for pain prevention process, which would be applicable only in exceptional cases, in a foreign legal doctrine suggestion to use it appears, based on the patient's explicit request, but in cases when the patient is no longer able to speak, deciding its application with the assistance of their representative [20]. Although efforts to improve legal regulation of palliative sedation are welcomed, such proposal can be accepted only partly.

Like euthanasia, palliative sedation affects the question of patient's continued existence and is the last resort to prevent pain and sufferings, therefore its practicing admissibility that would be based on a person's explicit request, not on the consent, must be declared 
to be rational and reasonable with a clause, unless it is intended to be applied as deep and continuous, resulting in unconsciousness. In turn, in cases of incapacity, the need to agree with the patient's representative before the use of palliative sedation, does not gain legal justification.

A representative of an incapacitated incurably ill person in the majority of cases will not be endowed with expertise in the medical field, therefore their decision that mainly is based on the physician's reasoning about the need to use palliative sedation acquires only a formal character without observation of the patient's interests and effectiveness of the procedure. Moreover, such agreement does not release the physician from the disciplinary responsibility, nor the potential criminal liability in cases where palliative sedation has been used either in bad faith, for example, covertly realizing any of the forms of euthanasia or unprofessionally performing their duties. Since the agreement between the physician, and incapacitated patient's representative on palliative sedation use does not guarantee the legality, in all cases when the patient is unable to express their will for such method, it shall be decided by a physician individually or also, if necessary, within the framework of the medical council, obliging only informatively notice the patient's representatives of intention to use the following method that does not find justification in the law, but in doctors ethics. An exception to this principle should be made only when the patient timely has secured their will in the legal document and their representative with the participation in the decision-making process only ensures its respect.

Condemning the cases when under deep and continuous palliative sedation actually is practiced euthanasia, as it is currently in the Netherlands, physicians' deliberate increasing of the medication dose that relieves the patient from sufferings, but inevitably leads to death, is considered as deviation and on theory level acquired the term "slow euthanasia". European Association for Palliative Care (EAPC) in 2009 issued recommended framework [21] on the use of sedation ethical, medical and legal preconditions within palliative care, recognizing it as an acceptable medical practice if it is used according to its purpose. However, the document emphasized that palliative sedation is the last resort to prevent pain, because it is related to a number of potential risks to the patient's life expectancy reduction.

According to the recommended framework, sedation is applicable only to the patients, in their last moments of life, with physical symptoms causing excruciating sufferings, and when due to the lack of time, there is no other palliative methods for prevention of sufferings that would not cause unacceptable side effects. However, its practicing sometimes is allowed in cases where the patient does not have only physical, but also psychological sufferings by giving an exhaustive list of this morbidity, namely incurable depression, anxiety, demoralization or existential sufferings [22]. Typical diseases causing fear of suffering, in which it can be applied to prevent palliative sedation, are psychosis, acute shortness of breath, pain and convulsions, but in emergency situations could be extended to massive bleeding, choking, etc.

A physician should always discuss with the patient the decision whether to use sedation, but in cases of their incapacity with their representative upon receipt of the consent. However, in the recommended framework it is encouraged, in cases of patient's incapacity, in the absence of pre-given guidance, not to impose a representative an obligation to make a decision, but its role is to get guidance for what the patient would have wanted from a physician in the following situations, while the responsibility for the medical decision is taken by medical personnel, as is apparent from the standards of care [23].

Unlike the Netherlands Medical Association "Palliative Sedation Guideline", the European Association of Palliative Care determines that continuous deep sedation may be applied only to the patient's life in the last stages, when life expectancy is measured in hours 
or a few days, without a mandatory requirement for its application up to the moment of death. Its application and duration always depends solely on medical indications, as well as the patient's will, and once with its help is achieved the primary task and there is no need for it to continue, it should be discontinued. Also, artificial hydration cessation is not a compulsory requirement, but its use or suspension is assessed in accordance with the wishes of the patient and medical indications [24]. The procedure provides for a mandatory negotiation with the patient of the possible negative consequences in cases of sedation, documenting its findings in detail. Also the same sedation process is a subject to mandatory documentation.

The use of medicines, their suspension, deep and continuous palliative sedation, as well as any other method used in medicine, associated with an increased risk of shortening the patient's life expectancy or causing death, in medical and legal doctrine has always been evaluated ambiguously. Over the centuries, the medical mission with the maximum permitted means to seek improvement of the patient's state of health or in the terminal phase release them from sufferings, is in conflict with the consequences, i.e., the probability to cause more damage than the expected benefits. Therefore, although in the ethical cross section between euthanasia and deep and continuous palliative sedation are there are no obvious differences, as they are the last resort in ending grievous pain and sufferings of incurably ill people care, which has no analogues in the world, in modern medicine and law doctrine it is fundamentally important to distinguish and apply medically correct delimitation of the action from the punishable crime in most countries of the world, i.e. homicide.

\section{Conclusions}

1. In the context of medical care of incurably ill people to alleviate pain and sufferings disposable medical products and methods, even if they contribute to the patient's life expectancy reduction or cause death, is not euthanasia, but from "double effect principle" consequential and accepted medical practice with condition that the performer acted on the basis of medical indications and in the absence of other alternatives, and its sole purpose was to release an incurably ill person from grievous pain and sufferings caused by disease, but the consequences, although were permitted, however, were undesirable. In all other cases, the doctor's actions, depending on the intention and motive, must either be accepted as intentional termination of the life of the patient, including an active voluntary, if the doctor's actions were based on a person's request, or in the absence cases, non-voluntary euthanasia or improper fulfilment of physician's professional duties for which criminal liability is provided. The double effect principle for the purpose of criminal law is not relevant to the general subject.

2. Deep and continuous palliative sedation is not the type of euthanasia, unless a physician who applies it, and the kind of it, i.e., until the elimination of grievous pain and sufferings caused by the disease, or death of the patient occurs, as well as maintaining or stopping the provision of patient's hydration, is primarily guided by the medical indications. In all other cases where that method actually provides a reduction of incurably ill patient's life expectancy, which is currently in the Netherlands, or is not justified by the need, physician's actions are not attributable to the levels of medical care and are assessed under criminal law as the intentional homicide of another person out of compassion or active euthanasia, and, depending on the circumstances in which it has taken place, either in the voluntary or non-voluntary manner. 
This work has been supported by the European Social Fund within the project "Support for Doctoral Studies at University of Latvia".

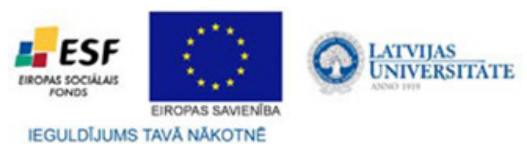

\section{References}

[1] M. Groenhuijsen, General Reports of the XVII ${ }^{\text {th }}$ Congress of the International Academy of Comparative Law, 938-940 (2007).

[2] S.W. Smith, End-of-Life Decisions in Medical Care: Principles and Policies for Regulating the Dying Process, 11 (2012).

[3] J. Legemaate, Physician-Assisted Death in Perspective. Assessing the Dutch Experience, 28 (2012).

[4] L. Amicorum, A. Kalmthout, Fervet Opus, 198 (2010).

[5] Guideline for Palliative Sedation. Committe on National Guideline for Palliative Sedation Royal Dutch Medical Association (KNMG). Ultrecht, The Netherlands. January 2009. Available: http://knmg. artsennet.nl/Publicaties/KNMGpubli catie-levenseinde/66978/Guideline-for-palliative-sedation-2009. htm[viewed on 28 January 2015].

[6] G. Hartog, Physician-Assisted Death in Perspective. Assessing the Dutch Experience, 368-369 (2012).

[7] L.W. Sumner, Assisted Death. A Study in Ethics and Law, 49 (2011).

[8] G. Williams, Medical Law Rewiev, 9, 41 (2001).

[9] P. Lewis, Assisted Dying and Legal Change, 6 (2007).

[10] G. Williams, Medical Law Rewiev, 9, 51 (2001).

[11] J. Griffiths, A. Bood, H. Weyers, Euthanasia and law un the Netherlands, 95 (1998).

[12] S.M. Behuniak, A.G. Svenson, Physician-Assisted Suicide. The Anatomy of a Constitutional Law Issue, 10 (2003).

[13] Ibid.

[14] E. Jackson, J. Keown, Debating Euthanasia (2012), p.16.

[15] G. Hartog, Physician-Assisted Death in Perspective. Assessing the Dutch Experience, 367 (2012).

[16] G. Hartog, Physician-Assisted Death in Perspective. Assessing the Dutch Experience, 367-368 (2012).

[17] J. Griffiths, H. Weyers, M. Adams, Euthanasia and Law in Europe, 66 (2008).

[18] G. Hartog, Physician-Assisted Death in Perspective. Assessing the Dutch Experience, 368 (2012).

[19] J. Legemaate, M.Verkerk, E. Wijlick, A. Graeff, European Journal of Health Law, 14, 66 (2007).

[20] J. Legemaate, M.Verkerk, E. Wijlick, A. Graeff, European Journal of Health Law, 14, 67-69 (2007).

[21] I.N. Cherny, L. Radbruch, Palliative Medicine, 23, 581 (2009).

[22] I.N. Cherny, L. Radbruch, Palliative Medicine, 23, 584 (2009).

[23] I.N. Cherny, L. Radbruch, Palliative Medicine, 23, 585 (2009).

[24] I.N. Cherny, L. Radbruch, Palliative Medicine, 23, 587 (2009). 